\title{
New Controllability Criteria for 3-phase 4-wire inverters applied to Shunt Active Power Filters
}

\author{
Manuel A. Perales, Luis Terrón, Juan A. Sánchez, Antonio de la Torre, Juan M. Carrasco, Leopoldo \\ G. Franquelo \\ Dept. de Ingeniería Electrónica. Escuela Superior de Ingenieros. Universidad de Sevilla \\ Camino de los Descubrimientos $\mathrm{s} / \mathrm{n}$ \\ Isla de la Cartuja. 41092-Sevilla . SPAIN \\ perales@gte.esi.us.es
}

\begin{abstract}
In Shunt Active Filter applications, the 3-phase 4wire topology is frequently used when dealing with unbalanced loads containing zero sequence components. A new design criteria for this topology is presented, based on the well known existing method for the 3-phase 3-wire system. Simulation and experimental results confirms the validity of this new criteria, providing an easy method for the design of the reactive elements involved in a Shunt Active Filter.
\end{abstract}

\section{INTRODUCTION}

Active Filters are a fast growing field in the power electronics area, due to the new regulations and standards on power quality. In particular, Shunt Active Filters are the most common structure, having nowadays a lot of practical implementations installed.

The most common structure in this field is based on the Current Controlled Voltage Source Inverter (CC-VSI). When designing this inverter, a special care have to be taken in the election of the reactive elements, that is, the capacitance and nominal voltage of the DC-Link and the value of the smoothing inductance.

When the load to be compensated is a 3-phase load, without neutral connection, a 3-phase 3-wire CC-VSI is commonly used. For this kind of inverters, a design criteria was developed long ago [1] that is very useful to select the correct values of this parameters, using the well known $\alpha-\beta$ transformation.

In this paper, an extension of this method is presented, covering the 3-phase 4-wire topology, also very employed in the Shunt Active Filters field [2] [3] [4]. In this case, the $\alpha-\beta$ transformation is not so useful, as it will be stated, because of the necessity of adding another component, the 0 axis, making the graphical analysis too much complicated.

In Section II, the 3-phase 4-wire topology is briefly presented and analysed, showing the RST- $\alpha \beta 0$ transformation of the inverter simplified equations. Then in Section III the controllability condition is defined, showing the difficulty on the assessment of this condition in $\alpha \beta 0$ axis. In Section IV a new change of axis is stated, called IMC transformation, that greatly simplifies the graphical analysis of the controllability problem. After that, in Section V simulation and experimental results are presented, that confirms the validity of the proposed method, and finally in Section VI some conclusions are extracted.

\section{3-PHASE 4-WIRE TOPOLOGY}

The 3-phase 4-wire topology is characterised by the connection of the neutral to the middle point of the DC-Link. In this situation, the three phases are independent of each other, and the current flowing for each leg depends only on the position of the associated switches and its phase voltage.

In fig. 1 a basic scheme of this topology is presented. It should be noted that the current is considered positive flowing out of the inverter.

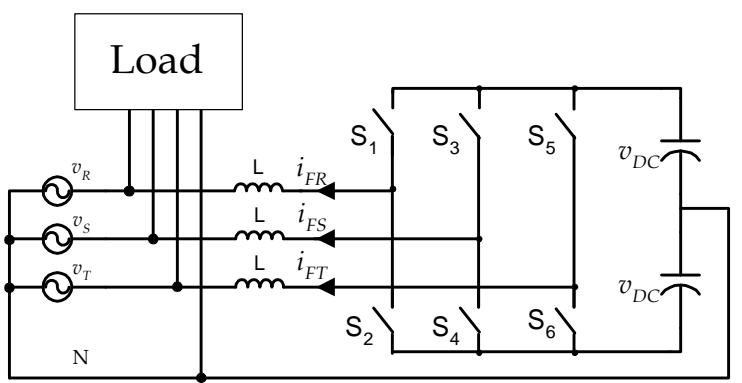

Fig 1. Basic scheme of a 3-phase 4-wire based Shunt Active Filter

So, the equations describing the behaviour of the phase currents could be expressed as (1)

$$
\mathbf{V}=\left[\begin{array}{c}
v_{R} \\
v_{S} \\
v_{T}
\end{array}\right] ; \mathbf{i}_{F}=\left[\begin{array}{c}
i_{F R} \\
i_{F S} \\
i_{F T}
\end{array}\right] ; \quad \mathbf{V}=-L \cdot \frac{d}{d t} \mathbf{i}_{F}+\mathbf{S}(k) \cdot v_{D C}
$$

Where $\mathbf{S}(\mathrm{k})$ is a vectorial discrete function depending on the state $(\mathrm{k})$ of the inverter, with the following values:

\begin{tabular}{clrrr}
\multicolumn{5}{c}{ TABLE I } \\
VALUES OF S(K) \\
State (k) & Switch. ON & S(k) & S(k) & S(k)T \\
\hline 0 & $\mathrm{~S}_{2}, \mathrm{~S}_{4}, \mathrm{~S}_{6}$ & -1 & -1 & -1 \\
1 & $\mathrm{~S}_{1}, \mathrm{~S}_{4}, \mathrm{~S}_{6}$ & 1 & -1 & -1 \\
2 & $\mathrm{~S}_{1}, \mathrm{~S}_{3}, \mathrm{~S}_{6}$ & 1 & 1 & -1 \\
3 & $\mathrm{~S}_{2}, \mathrm{~S}_{3}, \mathrm{~S}_{6}$ & -1 & 1 & -1 \\
4 & $\mathrm{~S}_{2}, \mathrm{~S}_{3}, \mathrm{~S}_{5}$ & -1 & 1 & 1 \\
5 & $\mathrm{~S}_{2}, \mathrm{~S}_{4}, \mathrm{~S}_{5}$ & -1 & -1 & 1 \\
6 & $\mathrm{~S}_{1}, \mathrm{~S}_{4}, \mathrm{~S}_{5}$ & 1 & -1 & 1 \\
7 & $\mathrm{~S}_{1}, \mathrm{~S}_{3}, \mathrm{~S}_{5}$ & 1 & 1 & 1 \\
\hline
\end{tabular}

As the three phase are decoupled, it is not possible to reduce it to a two component system, i.e. using the $\alpha \beta$ transformation. Instead, a three component reference system 
must be applied. If we use the $\alpha \beta 0$ transformation, defined by the matrix $M_{\alpha \beta 0}$ (2), the equations can be translated to (3):

$$
\begin{aligned}
& M_{\alpha \beta 0}=\sqrt{\frac{2}{3}}\left[\begin{array}{ccc}
1 & -1 / 2 & -1 / 2 \\
0 & \frac{\sqrt{3}}{2} & -\frac{\sqrt{3}}{2} \\
\frac{1}{\sqrt{2}} & \frac{1}{\sqrt{2}} & \frac{1}{\sqrt{2}}
\end{array}\right] \\
& L \frac{d \boldsymbol{i}_{\boldsymbol{F}}}{d t}=-U+V(k)
\end{aligned}
$$

Where the vectors $\boldsymbol{i}_{F}, U$ and $V(k)$ are derived from (4):

$$
\begin{gathered}
\boldsymbol{i}_{F}=\left[\begin{array}{l}
i_{F \alpha} \\
i_{F \beta} \\
i_{F 0}
\end{array}\right]=M_{\alpha \beta 0}\left[\begin{array}{c}
i_{F R} \\
i_{F S} \\
i_{F T}
\end{array}\right] ; U=\left[\begin{array}{l}
U_{\alpha} \\
U_{\beta} \\
U_{0}
\end{array}\right]=M_{\alpha \beta 0}\left[\begin{array}{l}
v_{R} \\
v_{S} \\
v_{T}
\end{array}\right] ; \\
V(k)=\left[\begin{array}{c}
V(k)_{\alpha} \\
V(k)_{\beta} \\
V(k)_{0}
\end{array}\right]=M_{\alpha \beta 0}\left[\begin{array}{c}
S(k)_{R} \\
S(k)_{S} \\
S(k)_{T}
\end{array}\right] \cdot V_{D C}
\end{gathered}
$$

It is important to note that all vectors $V(k)$ have a component on the 0 axis, as it can be seen in (5). That leads to two conclusions:

- Vectors 0 and 7 are not interchangeable, as it happened in the 3-wire topology.

- The hexagon formed for the vectors in the 3-wire topology is now a cube, rotated in the $\alpha \beta 0$ coordinates.

$$
\left[\begin{array}{l}
V(0) \\
V(1) \\
V(2) \\
V(3) \\
V(4) \\
V(5) \\
V(6) \\
V(7)
\end{array}\right]=\sqrt{\frac{2}{3}} \cdot V_{D C}\left[\begin{array}{rrr}
0 & 0 & -\frac{3}{\sqrt{2}} \\
2 & 0 & -\frac{1}{\sqrt{2}} \\
1 & \sqrt{3} & \frac{1}{\sqrt{2}} \\
-1 & \sqrt{3} & -\frac{1}{\sqrt{2}} \\
-2 & 0 & \frac{1}{\sqrt{2}} \\
-1 & -\sqrt{3} & -\frac{1}{\sqrt{2}} \\
1 & -\sqrt{3} & \frac{1}{\sqrt{2}} \\
0 & 0 & \frac{3}{\sqrt{2}}
\end{array}\right]
$$

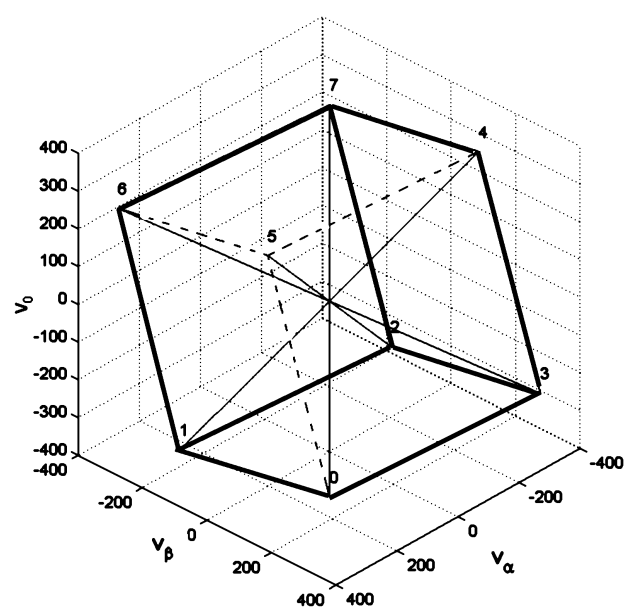

Fig 2. State vectors in $\alpha \beta 0$ coordinates, generating the controllability cube
This cube is the three dimensional equivalent to the well known two-dimensional hexagon produced by the vectors in the $\alpha \beta$ coordinates and will play an important role on the definition of the control capability of the inverter in following a certain current reference, so we called it controllability cube.

\section{CONTROLLABILITY CRITERIA IN $\alpha \beta 0$ COORDINATES}

Using the same technique as in [1] but for the three dimensional case, let us define $\boldsymbol{i}_{\boldsymbol{F}}$ as the current reference to be injected, and then the tracking error $\left(\Delta \boldsymbol{i}_{F}\right)$ could be expressed as:

$$
\Delta i_{F}=i{ }_{F}-i_{F}
$$

The condition to assure that the reference can be tracked is that the tracking error decreases with the time. So the controllability condition will be that the time derivative of this error is negative. Making this derivative, and using (3):

$$
L \frac{d \Delta \boldsymbol{i}_{\boldsymbol{F}}}{d t}=\left(L \frac{d \boldsymbol{i}^{*}{ }_{\boldsymbol{F}}}{d t}+U\right)-V(k)
$$

If we name $\boldsymbol{e}_{0}$ the term in parenthesis, (7) can be rewritten in the following form:

$$
\begin{aligned}
& \boldsymbol{e}_{0}=L \frac{d \boldsymbol{i}^{*}{ }_{\boldsymbol{F}}}{d t}+U \\
& L \frac{d \Delta \boldsymbol{i}_{\boldsymbol{F}}}{d t}=\boldsymbol{e}_{0}-V(k)
\end{aligned}
$$

Analysing (8) it is clear that the controllability depends on which vector is greater, $\boldsymbol{e}_{0}$ or $V(k)$. To ensure the controllability in the three phases simultaneously, the three components of the vector $\boldsymbol{e}_{\boldsymbol{0}}$ should be smaller than those of the $V(k)$ vector.

If a Space Vector modulation is implemented, $V(k)$ will represent the voltage vector of the inverter, and it will be included on the previously defined controllability cube. Then, the controllability criteria could be expressed as:

For given values of $V_{D C}$ and $L$, the so defined inverter will be capable of tracking a certain current reference only if the vector $\boldsymbol{e}_{\boldsymbol{0}}$ defined in (8) is contained on the inside of the controllability cube described by the vectors $V(k)$.

It is important to notice that, even in the case that the load has no zero sequence component, is not applicable the two dimensional criteria, because the intersection of the controllability cube with the $\alpha \beta$ plane is not equal to the hexagon described by the projection of the state vectors over the $\alpha \beta$ plane. This projection is equal to the hexagon used in [1], but the intersection of the cube with the $\alpha \beta$ plane, where should be located $\boldsymbol{e}_{\boldsymbol{0}}$ if it do not has zero sequence to ensure controllability, is an hexagon smaller than that, with its vertices in the middle of the sides of the first, as it is shown in fig. 3 . 


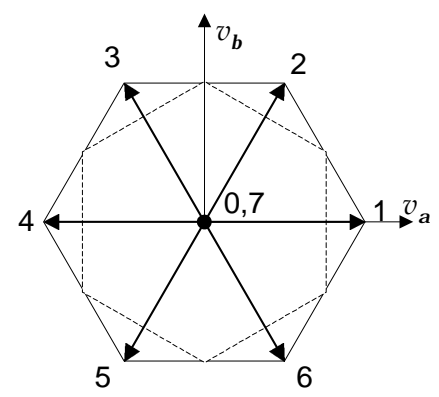

Fig 3. Controllability hexagon in the two dimensional case (solid) and intersection of controllability cube with the $\alpha \beta$ plane(dashed)

\section{NEW CONTROLLABILITY CRITERIA IN IMC COORDINATES}

\section{A. IMC transformation}

The complexity of the application of this new controllability criteria lays on the difficulty to determine whether $\boldsymbol{e}_{\boldsymbol{0}}$ is contained or not in the controllability cube, as it is rotated from the reference system.

To overcome this problem, a new transformation is proposed, called IMC transformation (whose name comes from Inverse of Matrix of Controllability), stated as:

- The new coordinates will be defined by the unitary vectors $v_{l}, v_{M}, v_{C}$, in the direction of $V(0)-V(5), V(4)-V(5)$ and $V(6)-V(5)$ respectively.

- The centre of the reference system will be placed on $V(5)$.

- The axis will be scaled down so the vectors $V(0)-V(5)$, $V(4)-V(5)$ and $V(6)-V(5)$ will have unitary modulus.

To make this coordinates change, it is useful to define the Matrix of Controllability (MC), constructed by columns with the vectors $V(0)-V(5), V(4)-V(5)$ and $V(6)-V(5)$ expressed in $\alpha \beta 0$ coordinates:

$$
\left.\begin{array}{l}
V(0)-V(5)=V_{D C}\left(\sqrt{\frac{2}{3}}, \sqrt{2},-\frac{2}{\sqrt{3}}\right) \\
V(4)-V(5)=V_{D C}\left(\sqrt{\frac{2}{3}}, \sqrt{2}, \frac{2}{\sqrt{3}}\right) \\
V(6)-V(5)=V_{D C}\left(2 \sqrt{\frac{2}{3}}, 0, \frac{2}{\sqrt{3}}\right)
\end{array}\right\} \Rightarrow
$$

Note that the columns of this matrix represents three orthogonal vectors placed in three sides of the controllability cube, and the three of them have the same modulo: $2 \mathrm{~V}_{\mathrm{DC}}$.

Now, to express a generic vector $\mathrm{Y}_{\alpha \beta 0}$ in the IMC reference system, it is only necessary to subtract from it the $V(5)$ vector, in $\alpha \beta 0$ coordinates, and then pre-multiply this by the inverse of the matrix of controllability, resulting:

$$
Y_{I M C}=M C^{-1}\left(Y_{\alpha \beta 0}-V(5)_{\alpha \beta 0}\right)
$$

Operating with (10), (9) and (5), the change of coordinates from $\alpha \beta 0$ to IMC can be stated finally as:

$$
\left[\begin{array}{c}
Y_{I} \\
Y_{M} \\
Y_{C}
\end{array}\right]=\frac{1}{V_{D C} \sqrt{3}}\left[\begin{array}{ccc}
\frac{1}{\sqrt{2}} & \sqrt{\frac{3}{2}} & -1 \\
-\frac{1}{\sqrt{2}} & \sqrt{\frac{3}{2}} & 1 \\
\sqrt{2} & 0 & 1
\end{array}\right] \cdot\left(\left[\begin{array}{c}
Y_{\alpha} \\
Y_{\beta} \\
Y_{0}
\end{array}\right]-\sqrt{\frac{2}{3}} V_{D C}\left[\begin{array}{c}
-1 \\
-\sqrt{3} \\
-\frac{1}{\sqrt{2}}
\end{array}\right]\right)
$$

\section{B. Controllability cube on IMC axis}

The virtue of this new coordinate system is that, on IMC axis, the controllability cube will be a unitary cube (with all its sides measuring 1) and with its faces parallel to the coordinate planes IM, IC and MC, as represented in fig.4

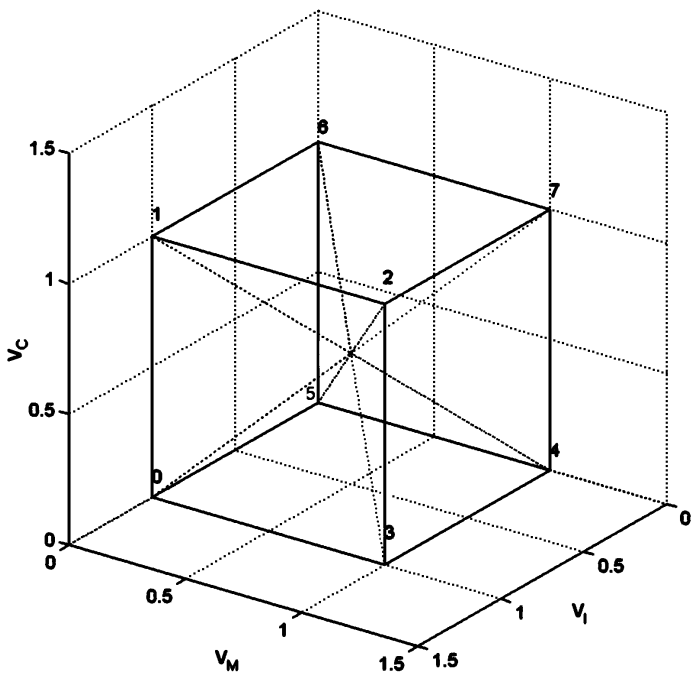

Fig 4. Controllability cube represented in IMC axis.

In this situation, the accomplishment of the controllability criteria can be very easily checked, only by testing that the IMC components of the $\boldsymbol{e}_{\boldsymbol{0}}$ vector remains between 0 and 1 always. If they do that, the vector will be contained in the controllability cube.

\section{Controllability Criteria in IMC coordinates}

Using the new coordinates transformation, the controllability criteria defined in III may be rewritten in this way:

For given values of $V_{D C}$ and $L$, the so defined inverter will be capable of tracking a certain current reference only if the IMC coordinates of the vector $\boldsymbol{e}_{0}$ defined in (8) remains between 0 and 1.

Note that $\boldsymbol{e}_{\boldsymbol{0}}$ depends not only on the current reference derivatives, but also on the value of the smoothing inductance, and even on the grid voltage. On the other hand, $V(k)$ depends on the DC-Link voltage. Consequently, for a given current reference and grid voltage, this two parameters $\left(V_{D C}\right.$ and $L$ ) could be adjusted to comply with this controllability criteria. 


\section{SIMULATION AND EXPERIMENTAL RESULTS}

To validate the criteria stated before, many simulation and experimental results were obtained. First a three phase unbalanced load, with zero sequence component, were used as load to compensate, doing extensive simulations to determine the optimal values for $\mathrm{V}_{\mathrm{DC}}$ and $\mathrm{L}$. Then , a three phase balanced load were simulated and compensated experimentally, offering the posibility of comparing the simulation and experimental results.

\section{A. Simulation with an unbalanced load}

A 3-phase 4-wire load with unbalance, zero sequence, current harmonics and reactive power was modelled from experimental measures of a real one. The wave shapes of the three phase currents and neutral current are drawn in fig. 5. It is noticeable the magnitude of the neutral current, related to the zero sequence component.

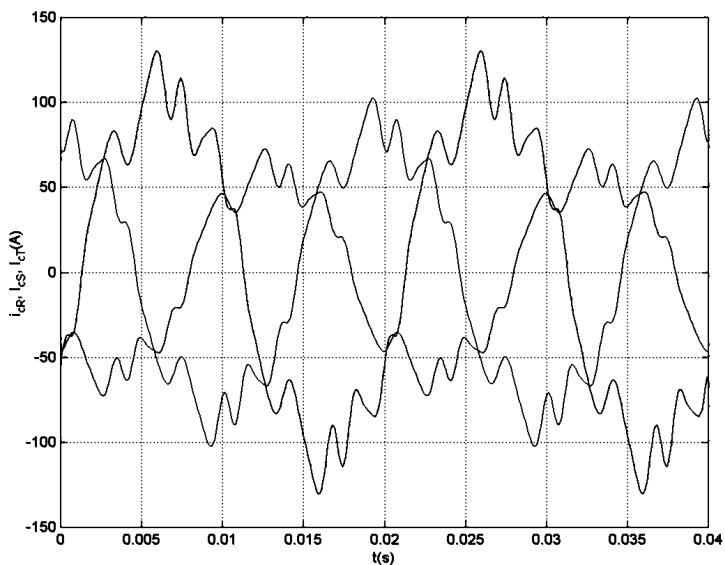

(a)

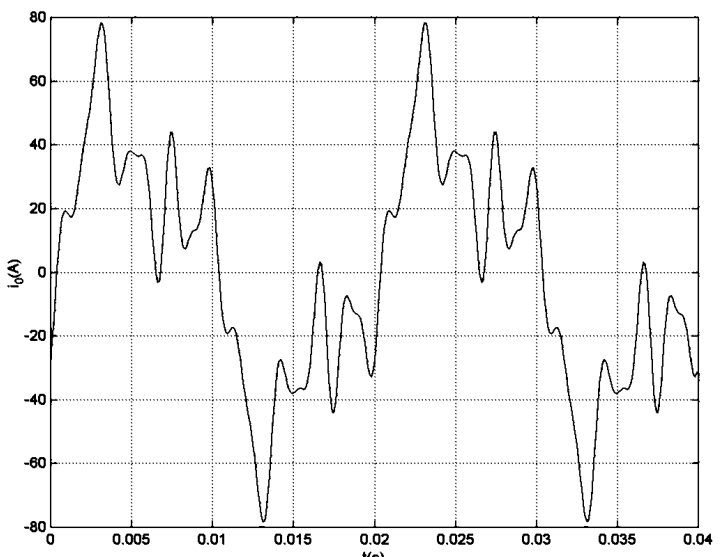

(b)

Fig 5. Unbalanced load simulated. (a) Load currents in R-S-T phases. (b) Neutral Current

Simulations were made calculating the vector $\boldsymbol{e}_{\boldsymbol{0}}$ needed to compensate adequately this load, for many values of $\mathrm{V}_{\mathrm{DC}}$ and
$L$. In fig. 6 , the three components of $\boldsymbol{e}_{0}$ in IMC coordinates are plotted for two particular conditions $\left(\mathrm{V}_{\mathrm{DC}}=250, L=1.5 \mathrm{mH}\right.$ and $\left.\mathrm{V}_{\mathrm{DC}}=300, L=1.2 \mathrm{mH}\right)$. It can be seen that in the first situation the controllability criteria will not be fulfilled, and it will in the second case.

Next, in fig. 7 , the optimal values for $\mathrm{V}_{\mathrm{DC}}$ depending on the value of $L$ is drawn. It can be seen that a quadratic relationship is almost followed (plotted in solid) by the calculated pairs (plotted as circles).

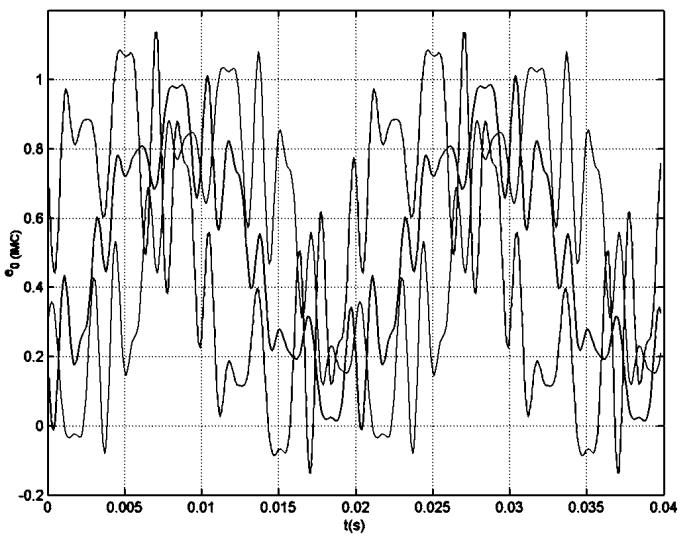

(a)

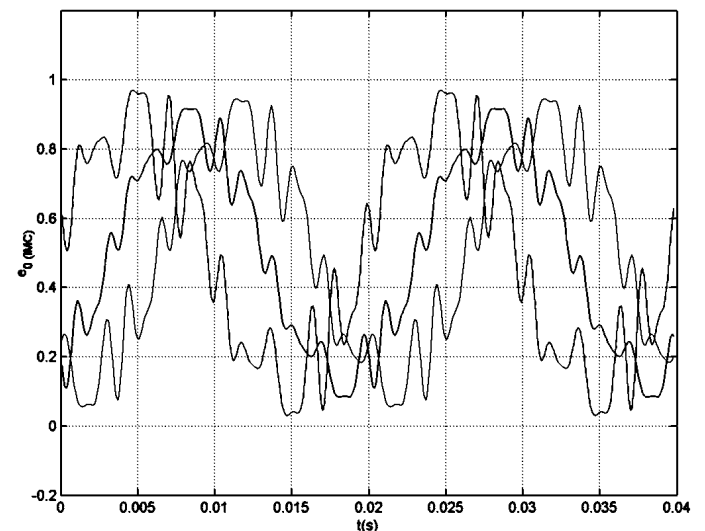

(b)

Fig 6. plot of $\boldsymbol{e}_{\boldsymbol{0}}$ in IMC coordinates. (a) $\mathrm{V}_{\mathrm{DC}}=300 \mathrm{~V}, L=2 \mathrm{mH}$. (b) $\mathrm{V}_{\mathrm{DC}}=300 \mathrm{~V}, L=1 \mathrm{mH}$

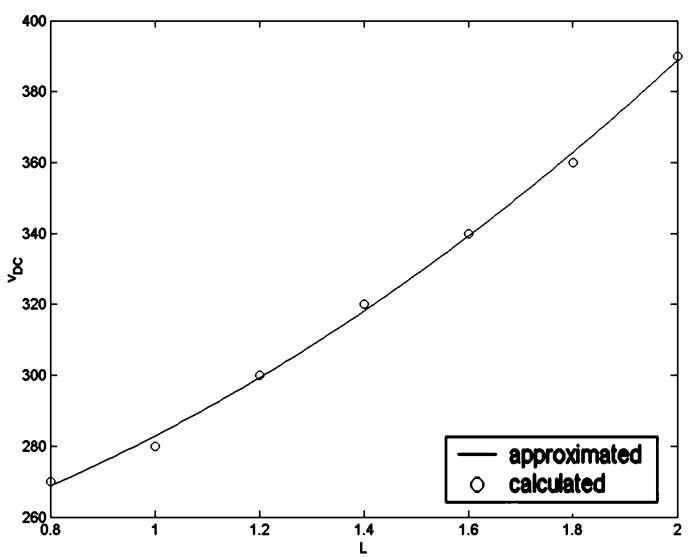

Fig 7. Optimal values for $\mathrm{V}_{\mathrm{DC}}$, for a given value of $L$. 
The curve obtained in fig. 7 gives us a design criteria for a Shunt Active Filter that might have to compensate this particular load: fixing the DC-Link voltage, due to switching or capacitor limitations, the inductance required can be calculated by means of this quadratic approximation.

\section{B. Simulations with a load without zero sequence}

Next, simulations of balanced load without zero sequence were performed, in order to prove that, in this case if a 3phase four-wire inverter is employed, the controllability hexagon applicable is the one defined in fig.3, and not the bigger one stated in [1].

The load simulated was modelled form laboratory measures, and is equal to the real one that will be compensated using an experimental Shunt Active Filter. The wave shape of the phase current is plotted in fig. 8 .

In fig. 9, the three components of $\boldsymbol{e}_{0}$ are plotted, for three values of $\mathrm{V}_{\mathrm{DC}}$ and the same value of $L$, showing that only the third case $\left(\mathrm{V}_{\mathrm{DC}}=300 \mathrm{~V}\right)$ complies with the controllability criteria. Then, in fig. 10 the polar representation of the $\alpha, \beta$ components of $\boldsymbol{e}_{\boldsymbol{0}}$ are plotted, for the same conditions as above.

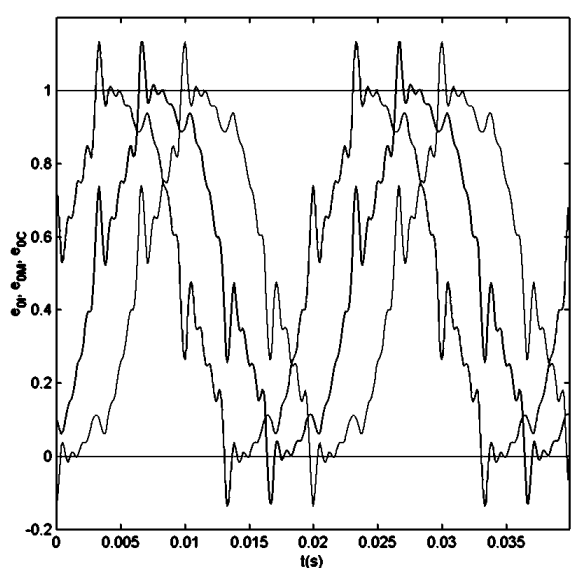

(a)

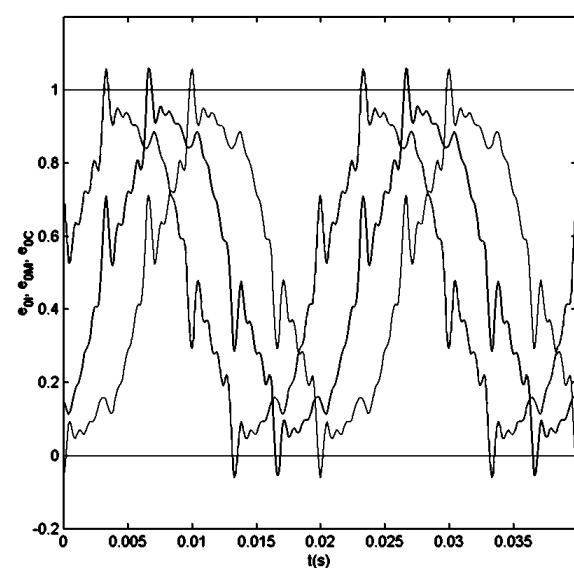

(b)

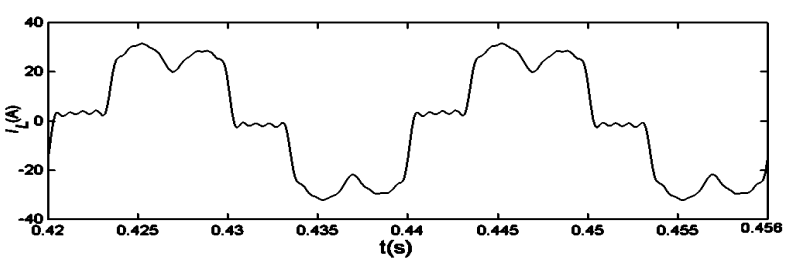

Fig 8. Load current used in simulations

Notice that, as $i_{L}$ has no zero sequence component, neither $i{ }_{F}$ will, so supposing that grid voltage has no zero sequence components, $\boldsymbol{e}_{\boldsymbol{0}}$ will be located on the $\alpha \beta$ plane. Then, the controllability criteria could be simplified to that $\boldsymbol{e}_{\boldsymbol{o}}(\alpha \beta)$ remains in the hexagon defined in fig. 3 . It is important to point out that, if the criteria defined for the 3-phase 3-wire case should be used, the situation represented in (b) should be considered as controllable erroneously, taking the outer hexagon (dashed) instead of the inner hexagon (solid) as the controllability hexagon.

Fig 9. Components of $\boldsymbol{e}_{0}$ in IMC coordinates. (a) $\mathrm{V}_{\mathrm{DC}}=225 \mathrm{~V}, L=2.4 \mathrm{mH}$. (b) $\mathrm{V}_{\mathrm{DC}}=250 \mathrm{~V}, L=2.4 \mathrm{mH}$. (c) $\mathrm{V}_{\mathrm{DC}}=300 \mathrm{~V}, L=2.4 \mathrm{mH}$

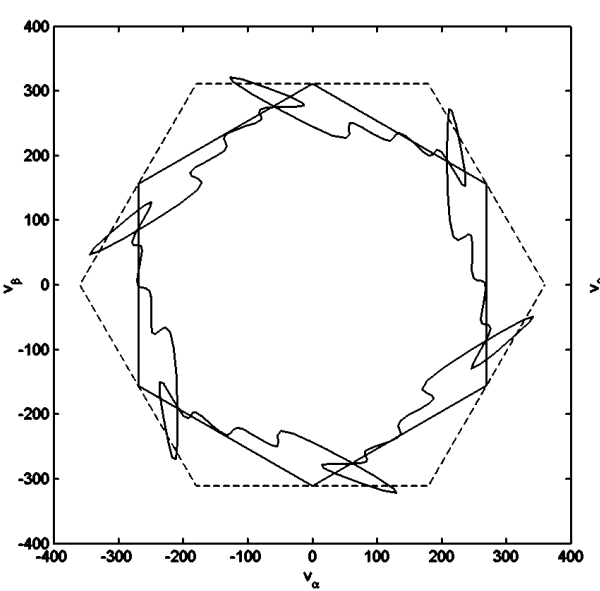

(a)

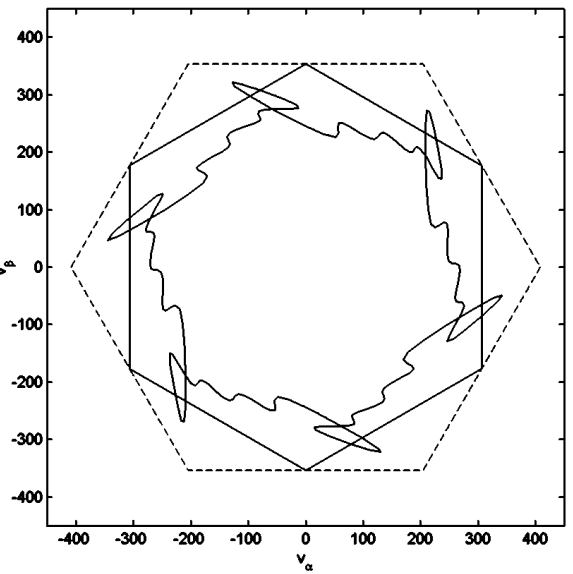

(b)

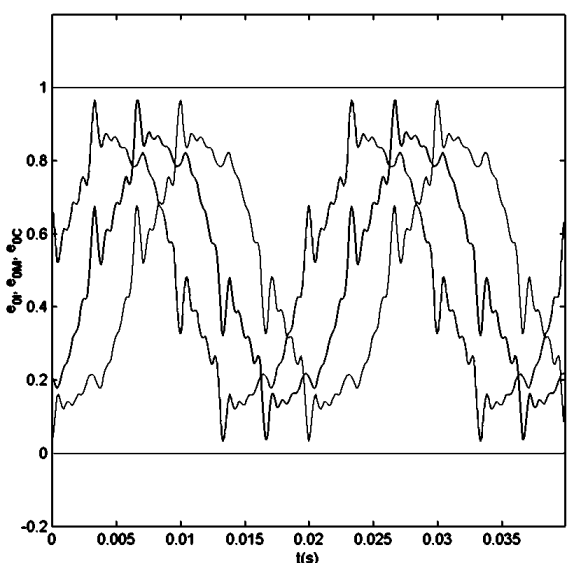

(c)

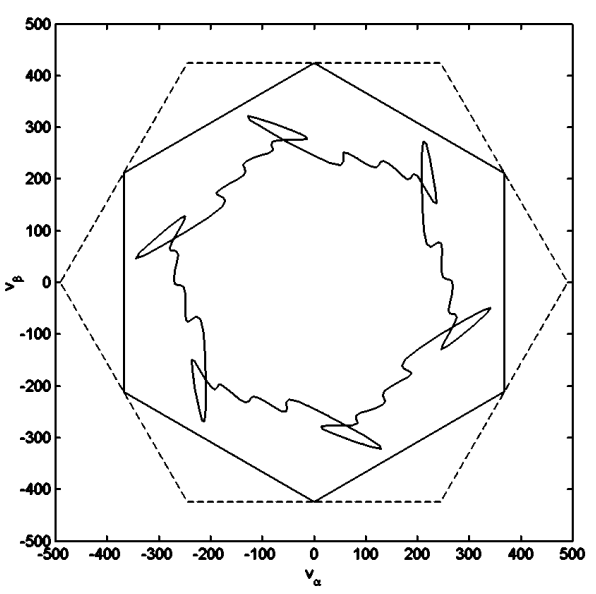

(c)

Fig 10. Polar representation of $\mathbf{e}_{0 \alpha}, \mathbf{e}_{0 \beta}$ with the new controllability hexagon (solid) and the 3-phase 3-wire case hexagon (dashed), for the same values as above. 


\section{Experimental results}

To validate the controllability criteria stated in this paper, experiments were made, using the same load than in the previous simulations.

For this experiments, an Active Power Filter developed by the authors was employed [5]. The control algorithm implemented was based on Self-Tuned Vector Filters [6], able to compensate current harmonics even in presence of a highly distorted voltage grid.

The most important parameters of the power system are shown in Table I .

\begin{tabular}{cc}
\multicolumn{2}{c}{ TABLE I. POWER SYSTEM PARAMETERS } \\
\hline Topology & 3-phase 4-wire \\
Voltage (phase-neutral) & $150 \mathrm{~V}$ \\
DC-Link capacity & $2 \times 13.2 \mathrm{mF}$ \\
DC-Link voltage (max) & $2 \times 350 \mathrm{~V}$ \\
Smoothing inductances & $2.4 \mathrm{mH}$ \\
Power switches & IGBT's (SKM300 GB 123 D) \\
Commutation frequency & $20 \mathrm{kHz}$ \\
Nominal power (load) & $12 \mathrm{~kW}$ \\
\hline
\end{tabular}

Finally, in fig. 11 experimental curves for the compensated current are shown. It can be observed easily that in the first and second cases a peak appears on the compensated current, due to the loose of controllability. This peak is greater in the first case $\left(\mathrm{V}_{\mathrm{DC}}=225 \mathrm{~V}\right)$, and do not appears in the third case $\left(\mathrm{V}_{\mathrm{DC}}=300 \mathrm{~V}\right)$

These results match precisely the predicted controllability of the system in terms of complying the controllability criteria stated, validating this way the proposed method.

\section{CONCLUSIONS}

The 3-phase 4-wire topology is widely used in the implementation of Shunt Active Filter solutions, having the ability to compensate unbalanced loads with zero sequence components. It is important therefore to have a design criteria, based on a controllability criteria in the same way than for the 3-phase 3-wire topology.

In this paper, a new controllability criteria is presented, as an extension of the two dimensional case to a three dimensional analysis.

By means of a transformation of coordinates, the criteria can be easily checked, allowing the use of it to estimate the correct values of the voltage of the DC-Link $\left(\mathrm{V}_{\mathrm{DC}}\right)$ and the smoothing inductance $(L)$.

The match between theoretical and experimental results confirms the validity of this technique, that should be used when a 3-phase 4-wire inverter is being designed for an Active Filter application, even if the load to compensate has not zero sequence components.

\section{REFERENCES}

[1]. H. Akagi, Y. Tsukamoto, A. Nabae. "Analysis and design of an active power filter using quad-series voltage source PWM converters," IEEE trans. on Industry applications Vol. 26 n ${ }^{\circ}$ 1, pp 93 -98. Jan-Feb. 1990

[2]. Amr M. A. Amin "Four-Wire Static Reactive Power Compensator" proceedings of the 26th Annual Conference of the IEEE Industrial Electronics Society IECON-2000, pp 960-964.

[3]. Nava-Segura, G. Mino-Aguilar "Four-Branches Inverter Based Active-Filter for Unbalanced 3-Phase 4-Wires Electrical Distribution Systems" proceedings of the Industry Applications Society conference IAS'2000.

[4]. C.A. Quinn, N. Mohan "Active Filtering of Harmonic Currents in 3-phase, 4-wire systems with 3-phase, single-phase nonlinear loads" Proceedings of the conference APEC'92, pp 829-836.

[5]. J.M. Carrasco, M. Perales, E. Galván, L. G. Franquelo, S. Gutiérrez, E.G. de Mendívil, "Dsp Control of an Active Power Line Conditioning System", proceedings of the conference EPE97 Vol. 3, pp. 1021-1026. 1997

[6]. M.A. Perales, J.L.Mora, L. Terrón, J.M. Carrasco, L.G. Franquelo "Transient Response and Dynamic Characterization of a New Active Filter Algorithm based on Self-tuned Vector filter", to be held in the Power Electronics Specialist Conference PESC02 on june,2002.

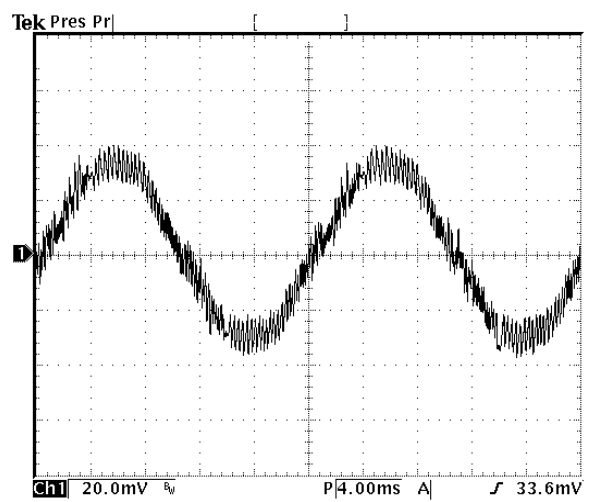

(a)

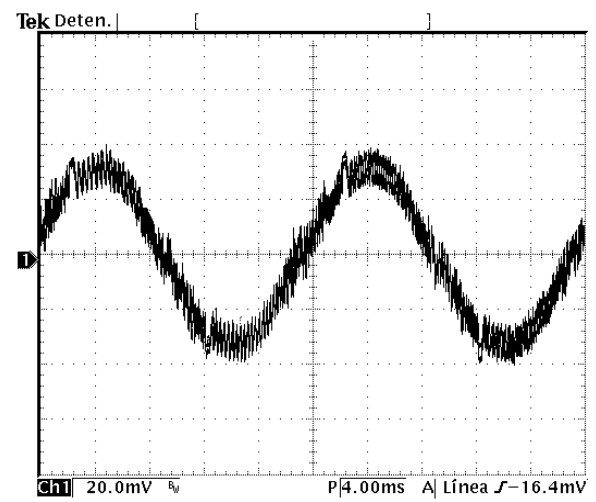

(b)

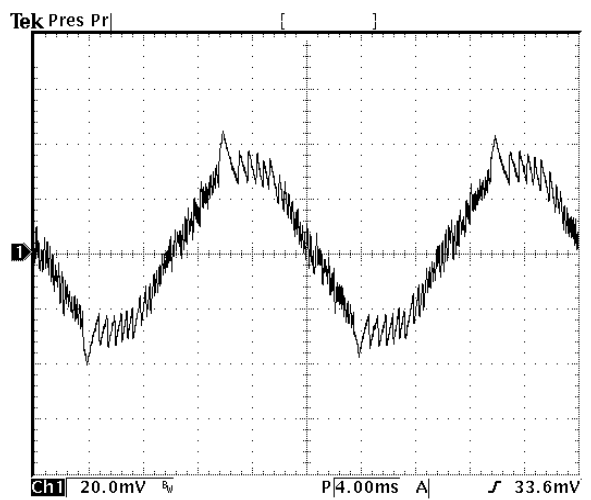

(c)

Fig 11. Experimental results. Compensated current for different values of $\mathrm{V}_{\mathrm{DC}}$, and $L=2.4 \mathrm{mH}$. (a) $\mathrm{V}_{\mathrm{DC}}=225 \mathrm{~V}$. (b) $\mathrm{V}_{\mathrm{DC}}=250 \mathrm{~V}$. (c) $\mathrm{V}_{\mathrm{DC}}=300 \mathrm{~V}$ 